\title{
The shifting landscape of Gender Identity and the situation in Sri Lanka
}

\author{
Dr A.S. Malalagama, Consultant Psychiatrist, Base Hospital Warakapola, Sri Lanka \\ Email: shakunthini@yahoo.com, (iD https://orcid.org/0000-0002-5507-9815
}

\section{Gender non conformity- a revolution}

Gender binarism is entrenched in cultures of Sri Lanka and most other Asian and Mediterranean countries. Gender non conformity was not heard of, a generation ago. Even today, majority are unaware of gender pluralism and non-conformity and use the words sex and gender interchangeably. Gender identity is an individual's perception and self-awareness of being male or female or a combination of male and female or neither male nor female. It is different from sex, sexual orientation or gender role. Gender identity lies in a spectrum as any other biological parameter such as height or weight $^{1}$. A recent survey of a thousand millennials found that half of them think gender is a spectrum. In the recent past, there are an increasing number of reports on youngsters questioning their gender identity. A person's gender is the complex interrelationship between three dimensions: Body (experience of our own body, how society interacts with and genders bodies), identity (our deeply held, internal sense of self as a male or a female) and expression (how we present our gender and how society perceive, interact with and shape our gender). Sexual identity: is in the perineum identified by external genitalia determined biologically.

Gender identity: is in the cerebrum identified by intellectual faculties determined culturally ${ }^{2}$.

Gender non conformity existed throughout history

Cultures with genders outside of a binary structure, or which identifies a third gender is found on every continent of the world throughout history ${ }^{1}$. Native Americans identified a third or fourth gender. In India and Pakistan intersex and transgender individuals are known as "hijra" and have

been identified as a third gender ${ }^{1,3}$. South Sulawesi, Indonesia, identifies five genders, including bissu, calalai', and calabai' ${ }^{1}$.

\section{Gender spectrum and transgenderism}

Gender non-conforming individuals are identified under the umbrella term, gender queer, which includes agender, androgyny, gender fluid and pangender ${ }^{4}$. Transgender refers to the broad spectrum of individuals who transiently or persistently identify with a gender different from their sex assigned at birth (American Psychiatric Association). Prevalence is 1 in 30000 natal males and 1 in 100000 natal females ${ }^{5}$.The prevalence of self-reported transgender identity in children, adolescents and adults is 0.5 to $1.3 \%{ }^{6}$.

Transgenderism should not be confused with intersex, transvestism and fetishistic transvestism, three conditions in which a person would assume a gender identity different to the expected. Intersex or hermaphrodites are born with both female and male sexual characteristics due to hormonal defects such as Congenital adrenal hyperplasia. Fetishistic transvestism is the wearing of clothes of the opposite sex principally to obtain sexual excitement ${ }^{7}$. Dualrole transvestism/ cross dressing is the wearing of clothes of the opposite sex for social and economic advantages or to enjoy the temporary experience of living in the opposite sex ${ }^{7}$. Hence, there are reasons other than transgenderism for a person to assume a different gender identity. Sometimes it may be difficult to differentiate between transsexuals, hermaphrodites and 
transvestites. Buhrich and McConaghy (1977) described different stages representing a continuum from transvestism to transsexuality $^{8}$. Some of the transvestites who start cross dressing before puberty are presenting with gender dysphoria in later life and request for gender reassignment procedures reflecting the overlap between the above conditions ${ }^{9}$.

Gender dysphoria, previously known as Gender Identity Disorders is a strong and persistent cross gender identification and a persistent discomfort with their sex or sense of inappropriateness in the gender role of that $\operatorname{sex}^{10}$. Gender dysphoria is classified as mental disorders and are therefore included in the DSM-IV and ICD-10 ${ }^{7}$.

Is gender dysphoria a mental illness?

This is a controversial issue. Gender dysphoria was classified as a mental disorder along with other disorders of gender identity based on historical theories surrounding its cause and because psychiatrists traditionally managed transgender patients ${ }^{11}$. But this classification has worsened the associated stigma.

LGBT activists have worked hard to declassify gender dysphoria from mental disorders. Denmark is the first country to be successful.

A study by Robles et al raises the question about the validity of classifying gender dysphoria as a mental disorder which concluded that "Distress and impairment which are considered essential characteristics of mental disorders, could be explained in the case of gender dysphoria by experiences of social rejection and violence rather than being inherent features of transgender identity"12.

Discrimination against gender non
conforming people

Report of the National Transgender Discrimination Survey in 2011 showed that gender non-conforming people face discrimination in every sphere of life including education, employment, family life, housing, identification documents, health care, insurance and justice system ${ }^{13}$.

In 2016, at least 22 deaths of transgender people in the US were due to fatal violence, the most ever recorded. Some of these cases involved clear anti-transgender bias. In others, the victim's transgender status may have put them at risk in other ways, such as forcing them into homelessness ${ }^{14}$.

LGBT people are more than twice as likely as Blacks or Jews and more than four times as likely as Muslims to be attacked in a violent hate crime ${ }^{15}$. Transgender people are 7 times more likely to experience physical violence when interacting with the police compared to cisgenders ${ }^{16}$.

The prevalence of suicide attempts is $41 \%$ among transgenders, which is more than 20 times the general population and is also higher than the $10-20 \%$ of lesbian, gay and bisexual adults who report ever attempting suicide $^{17}$.

There is a strong association between gender non-conformity and hate crime. As the definition of transphobia suggests, women who are perceived as masculine and men who are perceived as feminine, thus not conforming to normative standards of gender, are more likely to elicit an adverse reaction. This is backed by research suggesting that it is perceived gender transgression that may be at the root of both transphobic and homophobic hate crime ${ }^{18}$.

A survey by Human Rights watch in 4 districts in Sri Lanka among transgender and homosexual people between October 2015 and January 2016 reported legal discrimination towards this group, especially with regards to identification documents, health care and interaction with police ${ }^{19}$.

Even though there is no law specifically criminalizing transgender or intersex people, the offense of "cheat[ing] by personation" under section 399 of the Penal Code has been used to target transgender persons for arrest, 
assuming that appearing in a gender identity that is different from the sex assigned at birth has the malicious intent of cheating others ${ }^{19}$. The National Identity Card (NIC), the main identification document in Sri Lanka is issued on the information based on birth certificate. The sex assigned at birth stated in the NIC is different to the identified gender in transgendered, which leads to confusion among misinformed government and private officials who request the NIC to provide various services. Requests to change their name and gender in the NIC have been rejected by authorities depriving this group of basic human rights to apply for jobs, open bank accounts and obtain a passport/ driving licence and travel.

In the same report, male to female transgender people have reported several incidents of sexual abuse by police and arrests 19 .

Demand for transgendered in the sex industry

In Thailand and Philippines particularly transsexuals have a platform to speak out about their identity, their needs and even showcase themselves in international beauty pageants. In Thailand, where sex industry is booming, the demand for transsexuals or 'ladyboys' is high. Thailand has also become a hub for sex reassignment surgeries for an affordable cost, where surgeons run clinics under substandard conditions posing a risk to health of this population.

Even outside the sex industry, transgender women in Thailand may be more socially integrated than their peers elsewhere in the world, thanks in large part to sex trade ${ }^{21}$. In one study of 200 trans women in Thailand, subjects were found on average to be better educated and more affluent than the country as a whole. One Thai transgender woman who is the founder of TransFemale Association working for rights for transgender people, including public funding for genderreassignment surgery, won a provincial election in $2012^{21}$.
Transgender people worldwide are much more heavily involved in prostitution than the population at large, due to limited employment opportunities. Also, they engage in transactional sex in exchange for money for sex reassignment surgeries. In the U.S in 2011, $26 \%$ of transgender people had lost their jobs due to gender identity/expression and $11 \%$ had done sex work for income ${ }^{13}$.

Sexual health and risk for Sexually Transmitted Infections (STIs) among transgender population

Depression and anxiety, as well as illicit substance use to manage anticipatory anxiety related to transphobia, may contribute to high risk sexual behavior among transgender men ${ }^{22}$. Due to a power differential, transgender clients may not feel empowered to make decisions regarding drug use, condom use, and high risk sexual behavior, making them vulnerable to traumatic sexual experiences and STI exposure ${ }^{22}$.

Little research is done to understand risk for HIV and other STIs among transgender men who have sex with men and on transgender women ${ }^{22}$. Due to lack of funding, research has also focused mainly on HIV risk and prevention and not on other STIs or sexual health concerns, such as fertility or pregnancy ${ }^{22}$.

Transgender populations have higher rates of HIV infection than the general population ${ }^{22}$. Transactional sex may be a risk factor ${ }^{22}$. Little data exist on HIV among transgender men, likely due to much lower prevalence. However, evidence for HIV risk among transgender men who have sex with men is growing ${ }^{22}$.

HIV services for transgender people should address the specific biological, psychological, and social needs of this population. In line with national guidelines from the U.S. Centers for Disease Control (CDC) and the U.S. Preventive Services Task Force (USPSTF) that recommend universal screening for HIV, all transgender persons should be screened at 
least once for HIV. After initial screening, repeat screening is based on HIV risk assessment. Effective risk assessment requires the ability to obtain an accurate sexual history that includes anatomy-specific sexual behavior ${ }^{4}$.

The interest imparted by the Sri Lanka College of Sexual Health and HIV Medicine on sexual health of this group is commendable.

\section{Recent developments internationally}

In June 2016, the United Nations, Human rights Council adopted a resolution for protection against violence and discrimination based on sexual orientation and gender identity which reaffirms universality of rights $^{23}$.

In October 2015, a transgender rights activist in Nepal, became the first Nepali citizen to travel abroad carrying a passport marked " $O$ " for "other" instead of " $F$ " for "female" or M for "male." Nepal's Supreme Court recognized a third gender category for people who identify as neither male nor female and made clear that the ability to obtain documents bearing a third gender should be based on "self-feeling," and not the opinions of medical professionals or courts ${ }^{19}$.

In 2013, India's Supreme Court recognized transgender people constitute a third gender, declaring that this "is not a social or medical issue but a human rights issue" ${ }^{19}$. Similarly, in 2009, the Supreme Court in Pakistan recognized a third gender category ${ }^{19}$.

\section{Recent trends in Sri Lanka}

On September 17th 2017, a leading Sunday newspaper in Sri Lanka, reported a murder of a woman. Investigations revealed that she was actually a man dressed as a woman. Further, it was reported that the deceased was arrested for dressing as a woman in the past and has been warned by the police ${ }^{24}$. The journalist condemned cross-dressing and sexual relations outside heterosexuality illustrating the negative opinion of the general public.

Following complaints from three transgender women in Sri Lanka to National Human Rights Commission in 2015 after being rejected by Registrar General's Department to change their legal gender, a gender recognition certificate was proposed that would be accepted by all authorities for indicating gender on official documents, including the birth certificate, NIC and passport ${ }^{19}$.

In June 2016, the Ministry of Health issued a circular setting out guidance on issuing the "Gender Recognition Certificate". The draft certificate requires a psychiatrist to certify that a transgender person has been referred to "hormone therapy" and "necessary surgical treatments" and that "the person underwent gender transformation process." The psychiatrist must also certify that the person has completed "the social gender role transition as required". This process contradicts the international best practice which recommends that medical, surgical, or psychiatric treatment or diagnosis should not be required for legal gender change ${ }^{19}$. Nevertheless, this initiative can be considered as a first step towards identifying the rights and existence of the gender non binary group in a background of cis-heteronormative culture in Sri Lanka.

On Wednesday 15 November 2017 the UN reviewed Sri Lanka's record on human rights and made recommendations to amend sections 365 and 365A of the Penal Code, which targets Lesbian, Gay, Bisexual, Transgender, Intersex and Questioning (LGBTIQ) people in consensual, adult relationships. In response to issues raised, Deputy Solicitor General underlined the government's commitment to reforming Sri Lanka's penal code to ensure that it meets international human rights standards.

Sri Lankan representation in the 2016 World conference of the International Lesbian, Gay, Bisexual, Transgender and Intersex Association (ILGA) held in Bangkok and in the 
Miss International Queen, the world's largest and most prestigious beauty pageant for transgender women in 2011 held in China could be considered positive trends.

EQUAL ground is the only organization in Sri Lanka currently actively fighting for equal rights for the LGBTIQ community. Founded in 2004 it is also the oldest organization of this nature functioning in the country which advocates for the decriminalization of homosexual activity and the elimination of discrimination of individuals on the basis of their sexual orientation or gender identity.

Transgendered and people with gender dysphoria are being assessed and managed by Psychiatrists in Sri Lanka in sexual disorder clinics at present. But a specialized centre or clinics designated to cater this group is yet to be developed.

Studies on transgender/gender non conformity in Sri Lanka

The author contributed to a case series done by Ginige et al in 2011 which reveals the struggles of this minority group to come out of the closet and seek medical help for gender reassignment ${ }^{25}$. Another case series by the department of Sociology, University of Colombo has looked into how transgender individuals describe their gender identity and sexual orientation; the reactions of others towards their gender identity and sexual orientation and their strategies to cope with, avoid, or respond to the reactions they have experienced ${ }^{26}$.

Local studies are lacking. Both the Sri Lanka College of sexual Health and HIV Medicine and Sri Lanka College of Psychiatrists, the main two scientific bodies who has the expertise to shed light upon this group should collaborate and study gender non conforming population to help overcome the legislative hurdles and oppression by the cisheteronormative society.

\section{References}

1. Tharp AN. Gender Spectrum Theory.

2. Rao RN, Thombre A. Intercultural Communication: The Indian Context. 2015.

3. Senelick L. The Changing Room: Sex, Drag and Theatre. Routledge. 2000.

4. Madeline B. Guidelines for the Primary and Gender-Affirming Care of Transgender and Gender Nonbinary People, Center of Excellence for Transgender Health. 2016; June 17 (2).

5. Hoenig J, Kenna JC. The prevalence of transsexualism in England and Wales. British Journal of Psychiatry.1974; 124:181-90.

6. Zucker KJ. Epidemiology of Gender Dysphoria and Transgender Identity. Sex Health. DOI: 10.1071/SH17067.

7. World Health Organization. The ICD-10 Classification of Diseases, Clinical Descriptions and Diagnostic Guidelines.WHO.1992.

8. Buhrich, N., and McConaghy, N. The clinical syndromes of femmiphilic transvestism. Arch. Sex. Behav. 1977; 6: 397-412.

9. Doorn CD, Poortinga J, Verschoor AM. Cross-gender identity in transvestites and male transsexuals. Arch Sex Behav. 1994; Apr; 23(2):185-201.

10. Meyer W, Bockting W, Cohen-Kettenis P, et al. The Harry Benjamin International Gender Dysphoria Association's Standards of Care for Gender Identity Disorders, Sixth Version. Journal of Psychology \& Human Sexuality. 2002; 13: 1-30.

11. Pfafflin F, Ettner R, Monstrey S, et al. Mental Health Issues. Principles of Transgender Medicine and Surgery. Haworth Press.2007.

12. Robles R, Fresán A, Vega-Ramírez $H$, Cruz-Islas J, Rodríguez-Pérez $V$, Domínguez-Martínez $T$, Reed GM.Removing transgender identity from the classification of mental disorders: a Mexican field study for ICD-11. Lancet Psychiatry. 2016; Sep; 3(9):850-9.

13. Grant JM, Mottet LA, Tanis J. Injustice at every turn: a report of the National Transgender Discrimination Survey. The National Gay and Lesbian Task Force and the National Center for Transgender Equality. 2011.

14. www.hrc.org/resources/violence-against-thetransgender-community-in-2017.

15. www.splcenter.org/fighting-hate/intelligencereport/2015/crosshairs

16. https://avp.org/wp-content/uploads/2017/04/ ncavp transhvfactsheet.pdf

17. Haas AP, Rodgers PL, Herman JL. Suicide Attempts among Transgender and Gender Non-Conforming Adults-Findings of the National Transgender Discrimination Survey. American Foundation for Suicide Prevention, Williams Institute, UCLA School of Law.2014.

18. Turner L, Whittle S, Combs R. Transphobic Hate Crime in the European Union. Research Project Sponsored by ILGA-Europe and Press for Change.2009. 
19. Human Rights Watch. All Five Fingers Are Not the Same" Discrimination on Grounds of Gender Identity and Sexual Orientation in Sri Lanka.2016.

20. https://www.cityweekly.net/utah/transsiam/Content?oid=2927335.

21. https://www.advocate.com/news/worldnews/2012/05/28/thai-transgender-activist-winsprovincial-election.

22. Keuroghlian AS et al. Sexual Health among Transgender People. A program of the Fenway Intsitute. National LGBT Health Education Centre.

23. http://www.un.org/ga/search/view_doc.asp?symb ol=A/HRC/32/L.2/Rev.1

24. Sunday Lankadeepa. Sep 17th 2017.

25. Ginige P, Rodrigo EK, Malalagama AS. "Case series on Transsexuals-A Sri Lankan experience" 33rd Annual Academic sessions of the Kandy Society of Medicine 2011.

26. Smaraweera HUS, Unseen Lives-Making Transgender Identities Visible: Dominant

Social Perceptions of the Transgender Community in Urban Sri Lanka. Faculty of Arts International Research Conference - 3rd \& 4th December,2015. 